\title{
Preservation of the Ability of Dissociated Quail Wing Bud Mesoderm to Elicit a Position-Related Differentiative Response
}

\author{
KATE M. STOCKER AND BRUCE M. CARLSON \\ Department of Biology (K.M.S., B.M.C.) and Department of Anatomy and Cell Biology (B.M.C.), University of Michigan, \\ Ann Arbor, Michigan 48109
}

\begin{abstract}
Previous studies showed that grafting wedges of fresh or cultured anterior quail wing mesoderm into posterior slits in chick wing buds resulted in the formation of supernumerary cartilage in a high percentage of cases. When anterior quail mesoderm, which had been dissociated into single cells and pelleted by centrifugation, was grafted into posterior slits of host chick wing buds, supernumerary rods or nodules of cartilage formed in $\mathbf{7 4 . 3 \%}$ of the cases. Few supernumerary skeletal structures formed following control operations in which pelleted dissociated anterior or posterior mesoderm was grafted into homologous locations in host chick wing buds. When pelleted, dissociated anterior mesoderm was cultured in vitro for 1 or 2 days prior to being implanted in posterior locations, the incidence of supernumerary cartilage formation increased to $95.5 \%$ and $93.8 \%$, respectively. The incidence of supernumerary cartilage formation following control orthotopic grafts of cultured mesoderm was $11.8 \%$ for 1-day and $31 \%$ for 2-day cultured anterior mesoderm; for 1- and 2-day cultured posterior mesoderm, the incidence of supernumerary cartilage formation was $20 \%$ and $41.7 \%$, respectively. Longer-term culture resulted in a substantial decrease in the percentage of supernumerary cartilage after anterior to posterior grafts and an increase in the incidence of supernumerary cartilage from control grafts. The results demonstrate that quail anterior wing bud mesodermal cells do not need to maintain constant contact with one another in order to retain the ability to form or stimulate the formation of supernumerary cartilage after being grafted into a posterior location in a host wing bud. This ability is retained when the pelleted dissociated mesoderm is cultured in vitro outside the limb field for at least 1 to 2 days.
\end{abstract}

\section{INTRODUCTION}

The experimental analysis of limb morphogenesis has relied heavily upon the production of abnormal or duplicated structures. Experimental manipulations leading to the formation of such abnormalities have been instrumental in the formation of a number of morphogenetic models, most of which have been based upon Wolpert's $(1969,1971)$ hypothesis of positional information.

The experiments reported here are based upon the observation that wedges of anterior wing mesoderm plus ectoderm (Iten and Murphy, 1980) or mesoderm alone (Carlson, 1984a) grafted into posterior slits of chick wing buds result in the formation of supernumerary struc- tures. Grafts of mesoderm plus ectoderm typically form recognizable supernumerary digits (Iten and Murphy, 1980; Javois and Iten, 1981), whereas nodules or rods of cartilage, but never digits, are seen after grafts of mesoderm alone.

The formation of graft-induced supernumerary structures has been incorporated into two major hypotheses that attempt to explain certain aspects of morphogenetic control within the developing limb bud. One is based upon the existence of a zone of polarizing activity (ZPA) located near the posterior margin of the limb bud (Saunders and Gasseling, 1968; Fallon and Crosby, 1977). When transplanted to the anterior margin of the wing bud, the ZPA induces the formation of duplicated wing structures. It has been postulated that the cells of the ZPA release a diffusible morphogen and that the mesodermal cells of the limb bud respond to different levels of the morphogen by forming different digits (Tickle et al., 1975).

The other hypothesis attributes the formation of supernumerary structures after grafts into various locations within the avian limb bud to local interactions between graft and host tissues that have sufficiently different positional values (Iten, 1982). This hypothesis is based upon the general principles of the polar coordinate model of morphogenetic control in the regenerating limb (French et al., 1976).

The experiments reported here are a continuation of an effort to understand more fully the nature of the interaction that leads to the formation of supernumerary structures when anterior and posterior cells are experimentally placed next to one another in a wing bud. Following the demonstration that wedge grafts of anterior wing mesoderm resulted in a more than $90 \%$ incidence of supernumerary cartilage in posterior regions of the host chick wing buds (Carlson, 1984a), it was shown that mesodermal wedges cultured in vitro, away from the continuing influences of the limb field, retain the positional properties that result in the formation of supernumerary cartilage after anterior-to-posterior grafting (Carlson, 1984b). As yet we know very little about these "positional properties" and can only say that this assay demonstrates a positionally related control of cartilage differentiation. We do not know the mechanism by which supernumerary cartilage differentiation is stimulated and cannot say how this phenome-

Address reprint requests to Dr. Bruce M. Carlson, Department of Anatomy and Cell Biology, 4622 Medical Science II Bldg., University of Michigan, Ann Arbor, MI 48109.

Received September 12, 1986. Accepted December 21, 1987. 


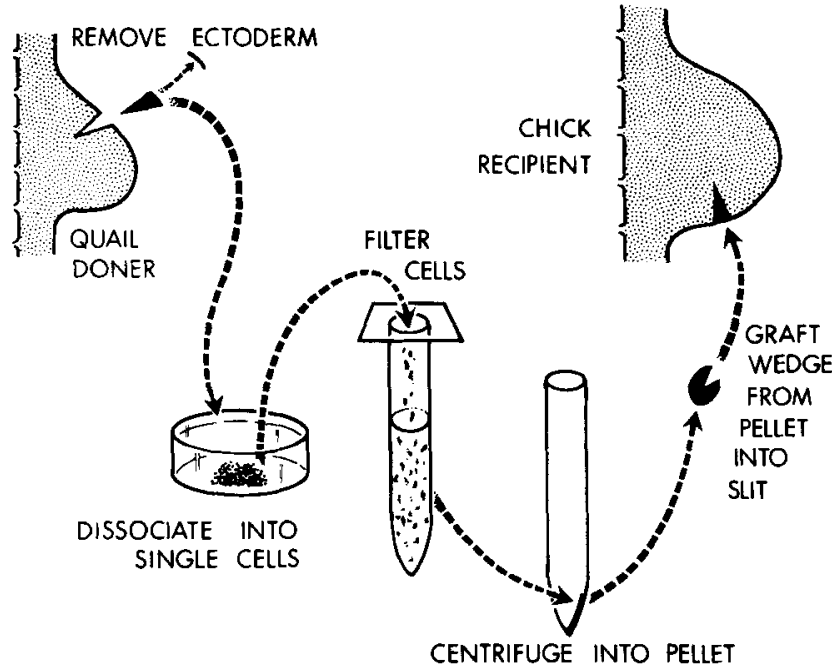

Fig. 1. Procedure for preparing grafts of pelleted, dissociated anterior wedges of quail wing bud mesoderm and grafting them into posterior slits of host chick wing buds.

non is related to the morphogenesis of recognizable supernumerary digits that occurs following grafts of mesoderm plus ectoderm.

The goal of the present experiment was to answer the question, "Must the cells of an anterior wing mesodermal wedge remain in constant contact with one another in order to retain their ability to form or stimulate the formation of supernumerary cartilage after being grafted into a posterior location in a host wing bud?" This question was approached by dissociating wedges of anterior quail wing bud mesoderm into single cells, pelleting them by centrifugation and then grafting pieces of the pellet into posterior slits in host chick wing buds. In other experiments, pelleted mesodermal cells were cultured in vitro before being grafted into a limb bud.

\section{MATERIALS AND METHODS Experimental Animals}

These experiments were conducted on embryos of a cross between White Leghorn and Rhode Island Red chickens (Gallus domesticus) (Dave's Eggs, Ann Arbor, MI) and on Japanese quail embryos (Coturnix coturnix japonica). Quail embryos provided donor tissue for all the experiments, while the chicken embryos served as hosts. The embryos were staged according to the criteria of Hamburger and Hamilton (1951). The eggs of both species were incubated at $37.5^{\circ} \mathrm{C}$ in a humidifed forcedair incubator before and after the operations.

\section{Surgical Procedures}

The basic experimental procedure involved removing wedges of anterior wing bud mesoderm from donor embryos, dissociating them into single cells, pelleting the cells by centrifugation, and then grafting a piece of the pellet into a posterior slit in the wing bud of a host embryo (Fig. 1). The wedges of anterior wing bud tissue (approximately $150-200 \mu \mathrm{m}$ on each side) were removed at the level of the junction of somites 16 and 17 from $H$. $H$ stage 20-23 quail embryos. The graft of pelleted dis- sociated cells was implanted into a posterior slit in the wing bud of a $\mathrm{H}-\mathrm{H}$ stage 20-23 chicken embryo (usually in the region of the prospective elbow but sometimes in more proximal prospective stylopodial regions in $\mathrm{H}-\mathrm{H}$ stage 20 hosts). Detailed descriptions of specific operations and controls will be presented with the results.

Two main experiments were performed. In the first, pieces of the pellet of dissociated cells were implanted immediately after they were prepared. In the second experiment, pieces of pelleted dissociated mesoderm were cultured in vitro for 1-4 days before being implanted into host wing buds. The latter results were compared with those for cultured intact wedges of mesoderm (Carlson, 1984b).

The host chicken embryos were prepared by withdrawing $2 \mathrm{ml}$ of albumen from each egg and cutting a window in the shell above the embryo. The membranes overlying the embryo were cut, and a slit was made in the wing bud with microscissors and tungsten needles. One or two drops of a penicillin-streptomycin solution (200 IU penicillin $/ \mathrm{ml}$ and $0.2 \mathrm{mg}$ streptomycin $/ \mathrm{ml}$ in Hanks' balanced salt solution) were added to each egg prior to the operation. After a piece of the cell pellet was implanted in the slit in a host wing bud, an additional one or two drops of penicillin-streptomycin solution were added to the egg. The wing was then traced with a camera lucida to record the position of the graft. The window in the egg was sealed with tape (Blenderm, 3M Co., St. Paul, MN), and the egg was returned to the incubator.

\section{Cell Dissociation Procedure}

The dissociated cell preparations were made by removing wedges of mesoderm plus ectoderm from right and left wing buds of donor quail embryos. The wedges were washed once in Dulbecco's phosphate-buffered saline (PBS, Gibco, Grand Island, NY) and three times in calcium- and magnesium-free Hanks' solution (Hanks' CMF, Gibco) at room temperature. The ectoderm was removed by incubating the wedges in $0.125 \%$ collagenase- $0.125 \%$ trypsin in Hanks CMF for $1 \mathrm{hr}$ at $4^{\circ} \mathrm{C}$. At the end of the incubation period, the wedges were washed three times in Hanks CMF containing 1\% new. born calf serum (Gibco). At this point, the ectoderm was often completely separated from the underlying mesoderm or could be picked off easily with fine needles.

Toward the end of the study, an alternate method for removing ectoderm was used because it provided a cleaner separation between the ectoderm and mesoderm and did not alter the results. The wedges were first incubated in $2 \%$ trypsin-pancreatin for $10 \mathrm{~min}$ at $4^{\circ} \mathrm{C}$. They were then washed three times in Hanks' balanced salt solution (Hanks' BSS), and the ectoderm was picked off the mesoderm. Next, the mesoderm was washed three times in Hanks' CMF and incubated in $0.125 \%$ collagenase- $0.125 \%$ trypsin in Hanks' CMF for 30-40 min at $4^{\circ} \mathrm{C}$. The mesodermal wedges were washed three times in Hanks' CMF containing $1 \%$ newborn calf serum.

The mesodermal wedges obtained by the above methods were triturated through a fine-bore pipette. The resulting cell suspension was filtered through a single layer of Nitex cloth $(20-\mu \mathrm{m}$ pore size) to produce a suspension of approximately $98 \%$ single cells, with $2 \%$ twoor three-cell clumps. Cell viability was approximately $98 \%$, as assayed by the trypan blue exclusion method. 


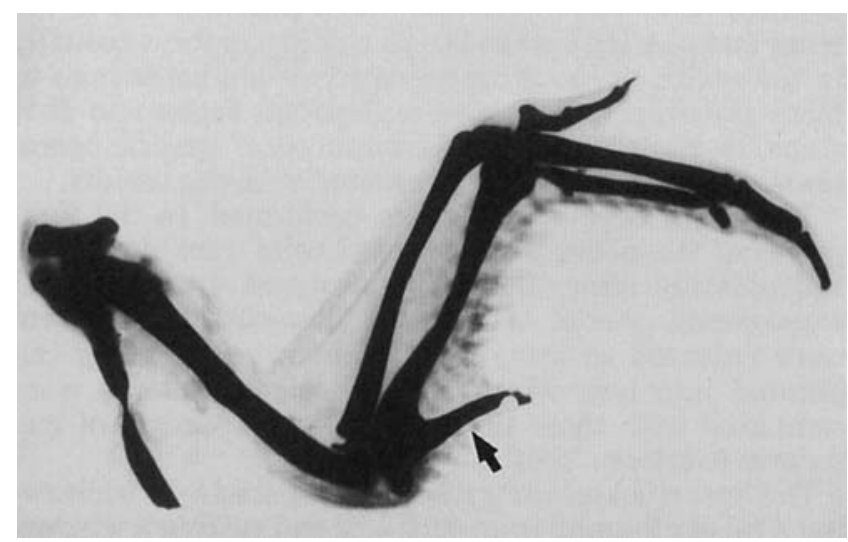

Fig. 2. Typical example of a supernumerary cartilaginous spike (arrow) formed after grafting a wedge of pelleted, dissociated anterior quail wing mesoderm into a posterior slit of a host chick wing bud. Wing removed 7 days after the operation and stained with Victoria blue $\mathbf{B}$.

The cell suspension was then centrifuged at approximately $500 \times \mathrm{g}$ for $3 \mathrm{~min}$, and the pellet was resuspended in Hanks' BSS containing 1\% newborn calf serum. The cells were centrifuged at approximately $\mathbf{5 0 0}$ $\times \mathrm{g}$ for $10 \mathrm{~min}$, and the resultant pellet was placed in a dish of Hanks' BSS with $10 \%$ newborn calf serum. The pellet was cut into pieces (approximately 150-200 $\mu \mathrm{m}$ on each side) that were stained lightly with Nile blue sulfate and kept at room temperature until they were grafted or placed in culture.

\section{Tissue Culture}

Pieces of pelleted mesodermal cells that were to be cultured in vitro were placed on top of small pieces of Nuclepore filter $(0.6-\mu \mathrm{m}$ pore size) supported at the medium-air interface by fine-mesh stainless-steel screen platforms. Each platform was placed in a $35-\mathrm{mm}$ plastic culture dish of medium. The medium consisted of BME (diploid) containing Earle's salts and added glutamine (Gibco), with 10\% horse serum (Gibco) and 5\% chick embryo extract (Gibco). Penicillin (100 IU/ml), fungizone $(0.25 \mu \mathrm{g} / \mathrm{ml})$, and streptomycin $(100 \mu \mathrm{g} / \mathrm{ml})$ (Gibco Antibiotic/Antimycotic solution) were added to the medium. The tissues were cultured at $37.5^{\circ} \mathrm{C}$ in a highly humidified incubator with an atmosphere of $5 \%$ carbon dioxide and $95 \%$ air. For longer-term experiments, the medium was partially replaced on the third day. At the end of the culture period, the piece of filter with adhering mesenchyme was placed in PBS. The mesenchyme was lightly stained with Nile blue sulfate and was removed from the filter with tungsten needles.

The cultured mesoderm was grafted into a slit in a host chick wing bud. Because the wedges of cell pellet flattened into pancake-shaped masses during the culture period, the technique for implantation differed slightly from that used for grafts of fresh pellet pieces. First, the cultured mesoderm was often cut into one-half or one-third pieces with microscissors. Each fragment served as a complete graft and was usually folded in one-half or one-third as it was implanted into the slit. A few drops of penicillin-streptomycin solution were added to each egg; the windows were sealed with tape; and the eggs were returned to the incubator.

\section{Histological Procedures}

Pieces of cultured cell pellet were fixed at various time points (1, 2, 3, 4, and 5 days) in Kahle's fixative, embedded in paraffin, sectioned $(7 \mu \mathrm{m})$, and stained with alcian blue at $\mathrm{pH} 1.1$ (Lev and Spicer, 1964) and hematoxylin to demonstrate the extracellular matrix. Additional pieces of cultured cell pellet were fixed in Carnoy's solution for staining by the Feulgen technique and in Bouin's solution for staining with hematoxylin and eosin.

The embryos were removed from the eggs 7 to 8 days after the operation, and the wings were fixed by various methods. Some wings were fixed in buffered $10 \%$ formalin and were stained in $0.5 \%$ Victoria blue B dye for demonstration of the skeletal elements. Other wings were fixed in Bouin's solution, followed by Carnoy's fixative, and later stained by both the Victoria blue B and Feulgen methods. This technique (Carlson et al., 1986) allows examination of the skeletal elements in whole-mount preparations and subsequent examination of $7-\mu \mathrm{m}$ sections cut from the whole mounts. One can then determine the contribution of quail (graft) cells and host chick cells to any supernumerary structures identified in the whole mounts. This is done on the basis of the nuclear chromatin marker in quail cells that was described by LeDouarin (1973). Additional wings were fixed in Carnoy's solution at various time points $(3,6,8$, $12,16,24,28,41,72,96,120$, and $144 \mathrm{hr}$ ) after the operation, embedded in paraffin, serially sectioned ( 7 $\mu \mathrm{m})$, and stained by the Feulgen technique.

\section{Scanning Electron Microscopy}

A series of embryos was fixed in $1.5 \%$ glutaraldehyde in $0.2 \mathrm{M}$ sodium cacodylate buffer (plus $5 \mathrm{mM} \mathrm{CaCl}_{2}$ ) at various times after the operation $(2,6,8,17,19,24$, and

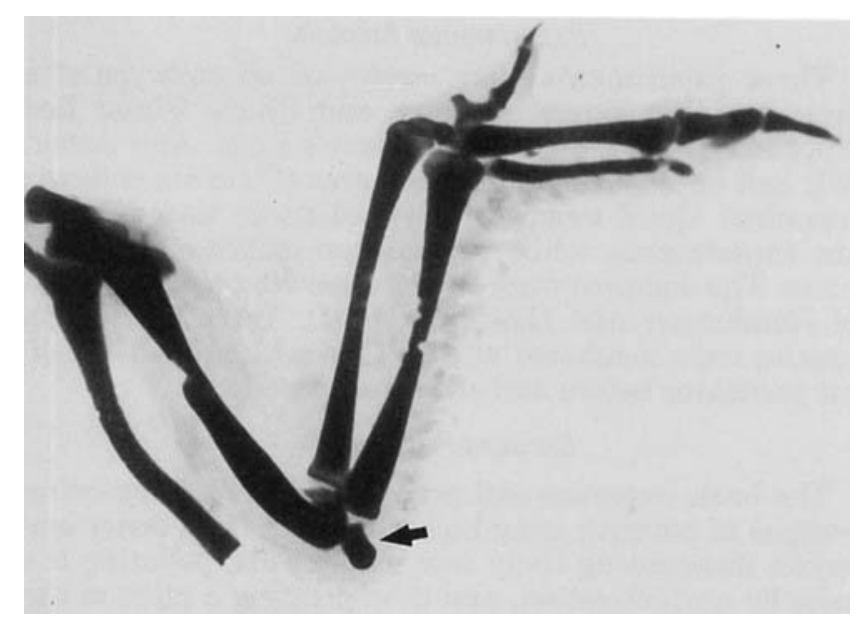

Fig. 3. Typical cartilaginous nodule (arrow) formed after grafting a wedge of pelleted, dissaciated anterior quail wing mesoderm into a posterior slit in a host chick wing bud. Wing removed 7 days after the operation and stained with Victoria blue B. 
TABLE 1. Formation of supernumerary (SN) structures after implanting freshly dissociated or cultured (1-4 days) pieces of dissociated anterior quail mesoderm (HH 20-23) into posterior slits in chick wing buds

\begin{tabular}{|c|c|c|c|c|c|c|c|}
\hline $\begin{array}{l}\text { Days in } \\
\text { culture }\end{array}$ & $\begin{array}{c}\text { No. } \\
\text { normal }\end{array}$ & $\begin{array}{l}\text { Cartilaginous } \\
\text { rods (No.) }\end{array}$ & $\begin{array}{l}\text { Cartilaginous } \\
\text { nodules (No.) }\end{array}$ & $\begin{array}{c}\text { Bent } \\
\text { humerus } \\
(\text { No. })\end{array}$ & $\begin{array}{c}\text { Other } \\
\text { anomaly } \\
\text { (No.) }\end{array}$ & $\begin{array}{c}\mathrm{SN} \\
\text { structures } \\
(\%)\end{array}$ & $\begin{array}{l}\text { Total } \\
\text { No. of } \\
\text { grafts }\end{array}$ \\
\hline 0 & 9 & 6 & 20 & 3 & 2 & 74.3 & 40 \\
\hline 1 & 1 & 7 & 14 & - & 2 & 95.5 & 24 \\
\hline 2 & 1 & 1 & 14 & 1 & 2 & 93.8 & 19 \\
\hline 3 & 20 & 3 & 27 & 1 & 5 & 60.0 & 56 \\
\hline 4 & 7 & - & 13 & 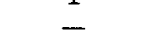 & 2 & 65.0 & 22 \\
\hline
\end{tabular}

${ }^{1}$ Bent humerus: these limbs were not included in the calculations of the percentage of SN structures.

${ }^{2}$ Other anomaly refers to limbs with amniotic bands or grossly distorted skeletons, or to embryos with ascites or other abnormalities. These limbs were not included in the calculations of the percentage of SN structures.

TABLE 2. Formation of supernumerary (SN) structures in control series for grafts of freshly dissociated and cultured pieces of dissociated quail mesoderm into chick wing buds

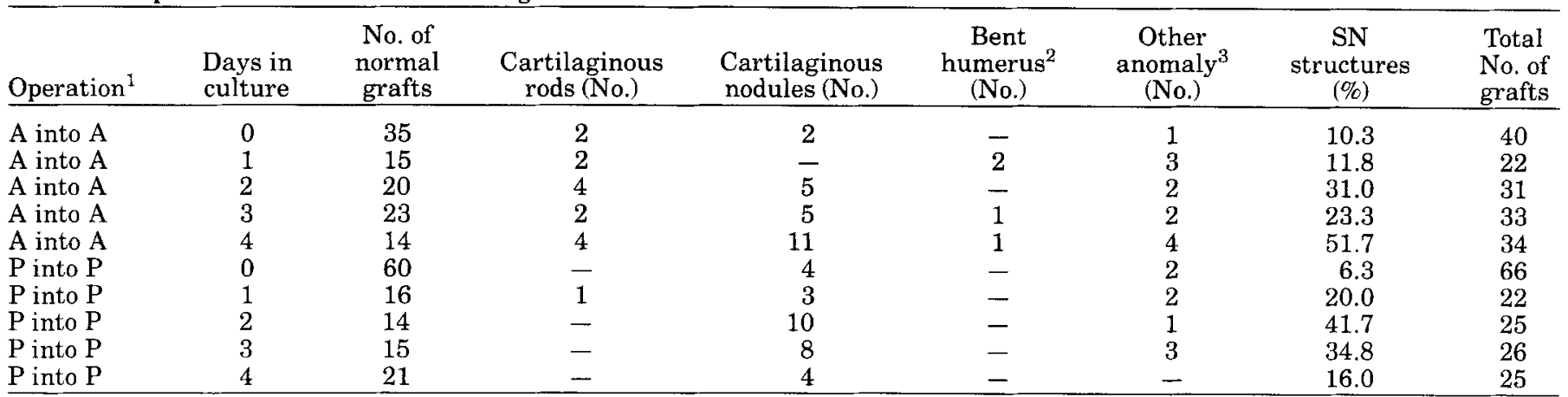

${ }^{1} \mathrm{~A}=$ anterior, $\mathrm{P}=$ posterior.

${ }^{2}$ Bent humerus: these limbs were not included in the calculations of the percentage of SN structures.

${ }^{3}$ Other anomaly refers to limbs with amniotic bands or grossly distorted skeletons, or to embryos with ascites or other abnormalities. These limbs were not included in the calculations of the percentage of SN structures.

$30 \mathrm{hr}$ ). The embryos were critical-point dried, sputtercoated with $20 \mathrm{~nm}$ of gold, and examined by scanning electron microscopy (ISI DS 130 scanning electron microscope).

\section{Measurements of Cartilaginous Structures}

A series of chick embryos in which pieces (150-200 $\mu \mathrm{m}$ on a side) of fresh intact anterior mesoderm from quail donors had been grafted to posterior slits in the host wings was killed 5 days after the operation. The wings were fixed and stained for cartilage with Victoria blue $B$ dye. The largest dimension of the supernumerary cartilaginous structure in each wing was measured in the whole mount preparations with a micrometer mounted in a dissecting microscope. These measurements were compared with the measurements of the cartilage nodules that developed in 5-day cultured anterior and posterior mesoderm (data for intact and pelleted dissociated cultures were combined). For the cultured tissues, the largest dimension of the cartilage nodule was measured in serial sections that had been stained with alcian blue and hematoxylin. A micrometer mounted in a light microscope was used.

\section{Statistics}

To compare the incidence of supernumerary cartilage formation among various groups, statistical tests of significance were done using the chi-square "fourfold" table (Hill, 1966). The Student's T-test (Hill, 1966) was used for comparisons of the size of the cartilage structures that developed in vivo following anterior-to-posterior grafts and during in vitro culture of anterior and posterior mesoderm.

\section{RESULTS \\ Results of Implant Experiments}

\section{Gross morphology of supernumerary structures}

In all the experiments reported here, any supernumerary (SN) skeletal structure demonstrated by Victoria 
blue B cartilage staining was scored as a positive result. The supernumerary structures usually fit into two categories: 1) cartilaginous rods or spikes of various lengths (Fig. 2) or 2) cartilaginous nodules or small protuberances from normal skeletal structures (Fig. 3).

\section{Grafts of fresh, pelleted dissociated mesoderm}

Supernumerary skeletal structures developed following $74.3 \%(26 / 35)$ of the operations in which fresh, pelleted dissociated anterior quail mesoderm was grafted into posterior slits in host wing buds. Fourteen of the supernumeraries were rods or large nodules of cartilage, while the rest were small nodules. Most of the supernumerary structures were found near the elbow or distal humerus, although a few were near the proximal humerus (Fig. 4, Tables 1, 2).

When fresh, pelleted dissociated anterior mesoderm was grafted into homologous anterior slits in host wings, $10.3 \%(4 / 39)$ of the wings developed with supernumerary structures. In two cases the supernumeraries consisted of small cartilaginous nodules, while in the other two cases a spur of cartilage projected from the distal humerus. The incidence of supernumerary formation was $6.3 \%(4 / 64)$ in the control posterior-to-posterior operations. In each case the supernumerary structure was a very small cartilage nodule near the elbow. The difference between the results of the control operations and the experimental operations was statistically significant $(P<.01)$

\section{Grafts of cultured, pelleted dissociated mesoderm}

Supernumerary skeletal structures resulted from $95.5 \%(21 / 22)$ of the grafts of 1-day cultured anterior mesoderm into posterior slits in host wing buds. Ten of the supernumerary structures were medium-sized or large cartilaginous nodules, usually in the elbow region, while four were small nodules and seven were rods or spurs of cartilage near the elbow or distal humerus (Fig. 5 , Tables 1,2 ).

Grafts of 2-day anterior mesoderm cultures produced 93.8\% (15/16) wings with supernumerary structures. Six of these supernumeraries were medium-large cartilaginous nodules located near the elbow; the rest consisted of small nodules near the elbow (eight cases) and one case of a spur near the distal humerus. After the grafts had been cultured for 3 days, the incidence of wings with supernumerary structures declined to $60 \%(30 / 50)$. These supernumeraries were predominantly small nodules of cartilage in the elbow or proximal ulna ( 25 cases), plus a few rods ( 3 cases) and medium-large nodules ( 2 cases). Grafts of anterior mesoderm cultured for 4 days produced wings with supernumerary structures in $65 \%$ $(13 / 20)$ of the cases. Most of these supernumeraries (12 cases) were small nodules in the elbow region, with one case of a large nodule.

Control grafts of 1-day cultured anterior mesoderm into homologous anterior slits in host chick wing buds resulted in $11.8 \%$ (2/17) wings with supernumeraries. Both were rods of cartilage attached to the mid or distal humerus. The incidence of wings with supernumerary structures was $20 \%$ (4/20) in posterior-to-posterior controls with mesoderm cultured for 1 day. The supernumeraries consisted of small nodules in the elbow and proximal ulna regions (three cases) and one spur at the wrist.

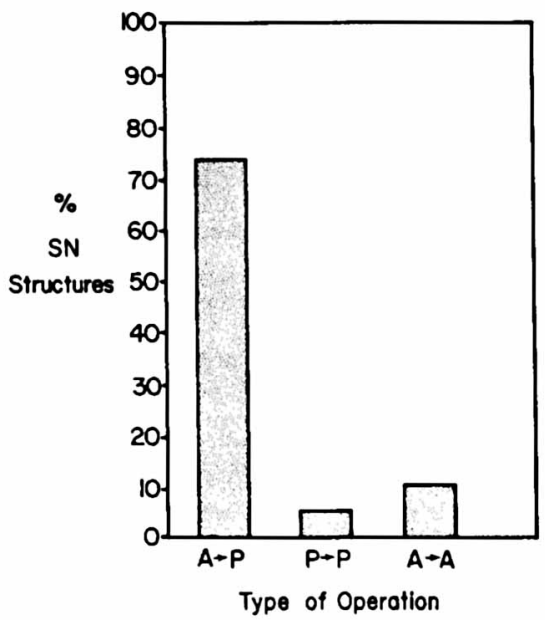

Fig. 4. Graph showing the percentage of supernumerary cartilage formed after grafting freshly pelleted, dissociated quail wing bud me-

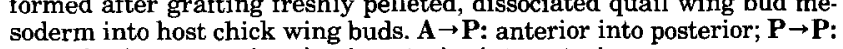
posterior into posterior; $\mathbf{A} \rightarrow \mathbf{A}$ : anterior into anterior.

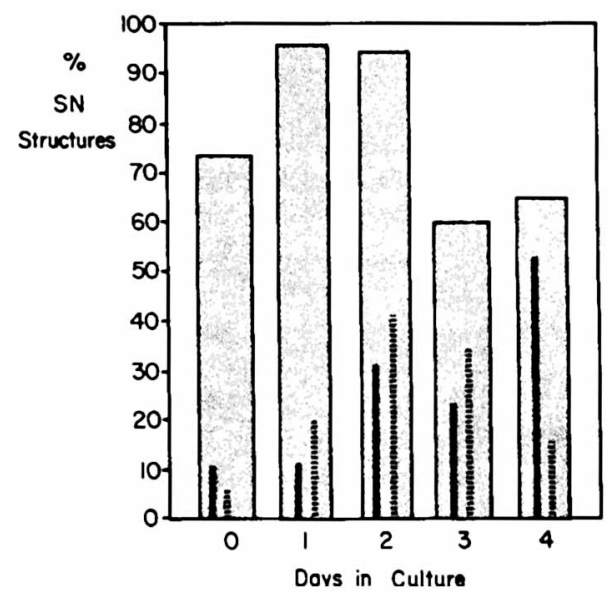

Fig. 5. Graph showing percentages of supernumerary cartilage formed after grafting cultured, pelleted, dissociated quail wing bud mesoderm into host chick wing buds. Shaded bars: anterior to posterior grafts; solid lines: anterior to anterior grafts; dashed lines: posterior to posterior grafts.

When anterior mesoderm was cultured for 2 days, the incidence of wings with supernumeraries rose to $31 \%(9 /$ 29) following anterior-to-anterior operations (4 rods, 2 large nodules, and 3 small nodules at various positions beside the humerus). Posterior-to-posterior grafts of 2day cultured mesoderm produced wings with supernumeraries in $41.7 \%(10 / 24)$ of the cases. Most of these (eight cases) were small nodules near the elbow or proximal ulna, with two cases of a thin sliver of cartilage parallel to the mid ulna region. For the 1- and 2-day control operations, the incidence of supernumerary cartilage was significantly lower $(P<.01)$ than for the corresponding experimental operations.

After 3 days in culture, anterior mesoderm grafted into homologous anterior slits resulted in $23.3 \%(7 / 30)$ wings with supernumerary cartilage. The supernumer- 
aries consisted of rods extending from the humerus (two cases) or small nodules (five cases). In two cases the nodule was in the anterior wing web. The incidence of supernumerary formation following 3-day posterior-toposterior operations was $34.8 \%(8 / 23)$. All the supernumeraries were small cartilage nodules near the elbow or proximal ulna. Although the percentage of supernumeraries was significantly lower for the control anterior-toanterior operations than for the 3-day experimental op-

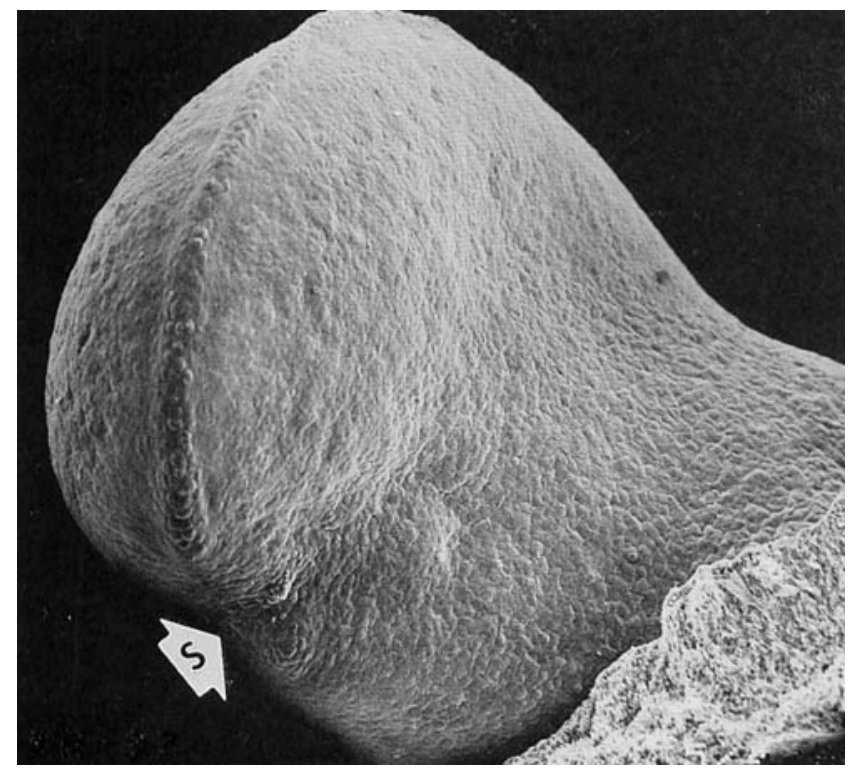

Fig. 6. Scanning electron micrograph showing the discontinuous apical ectodermal ridge of a chick wing bud fixed $24 \mathrm{hr}$ after a wedge of pelleted, dissociated anterior quail wing bud mesoderm was implanted into a posterior slit. $S=$ healed-over implantation site. $\times 106$.

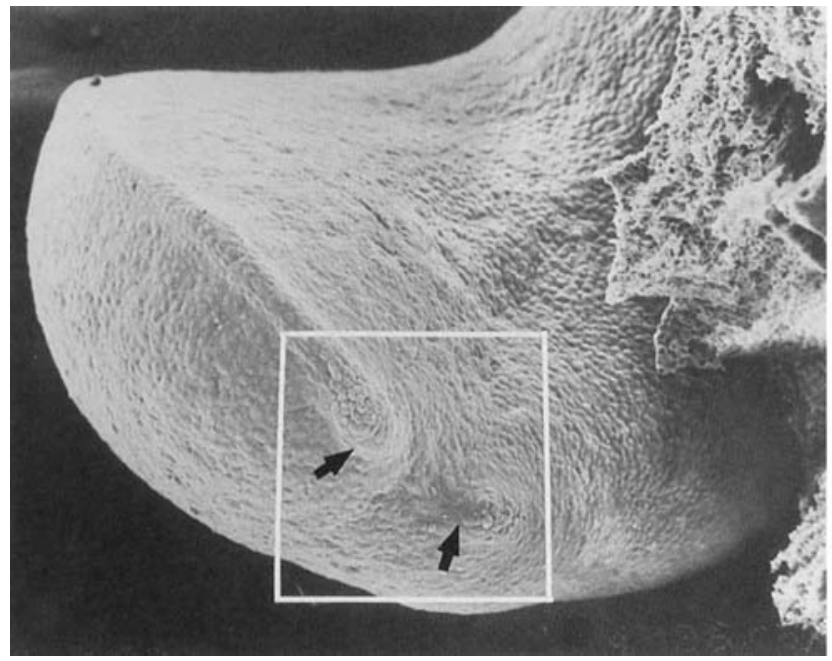

Fig. 7. At left, a scanning electron micrograph of a discontinuous apical ectodermal ridge (AER) (arrows) on a wing bud fixed $33 \mathrm{hr}$ after a wedge of pelleted, dissociated posterior quail wing bud mesoderm erations $(P<.01)$, the difference between the 3 -day posterior-to-posterior operations and the experimental operations was not statistically significant.

Anterior mesoderm cultured for 4 days and grafted into homologous anterior slits in host wing buds produced wings with supernumerary structures in $51.7 \%$ $(15 / 29)$ of the cases. Ten of these were small nodules beside the humerus; in one case, a nodule developed in the anterior wing web. The remaining cases consisted of rods or spurs of cartilage attached to the humerus (four cases) and one large nodule in the elbow. Posterior-toposterior operations with mesoderm cultured for 4 days resulted in $16 \%$ (4/25) wings with supernumeraries, all of which were small nodules in the elbow or beside the distal humerus. The incidence of supernumerary cartilage formation was significantly lower $(P<.01)$ for the posterior-to-posterior operations than for the 4-day experimental operations, but the results for the anteriorto-anterior controls did not differ significantly $(P<.01)$ from the experimental results.

\section{Reaction of Chick Wing Bud to Implants of Freshly Dissociated Mesoderm}

Serial cross sections and scanning electron micrographs of operated embryos were examined in order to determine 1) the time course of ectodermal healing over the graft, 2) if the apical ectodermal ridge (AER) is reestablished over the graft, and 3) whether the implanted quail cells remain cohesive or if they are dispersed within the host limb.

\section{Ectodermal healing reactions}

Ectodermal healing was complete (8/19 embryos) or nearly complete (11/19 embryos) within a day after im. planting dissociated and pelleted anterior mesoderm into posterior slits. Scanning electron microscopy examination of seven wing buds from 17 to $24 \mathrm{hr}$ after grafting revealed a discontinuity in the AER over the graft site in most embryos that had undergone complete healing

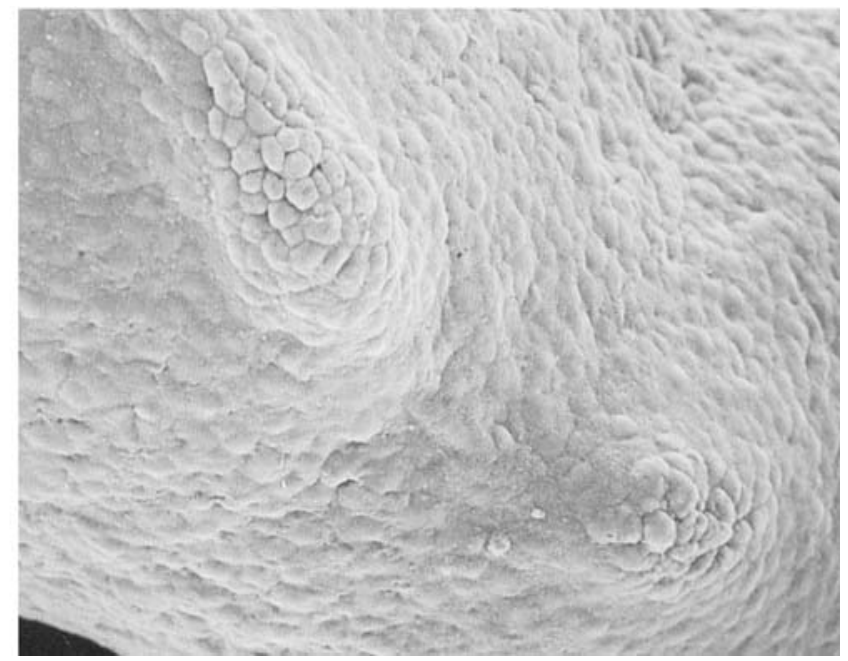

was implanted into a posterior slit. Magnification, $\times 105$. At right, high-power view $(\times 316)$ of the discontinuity in the AER. 

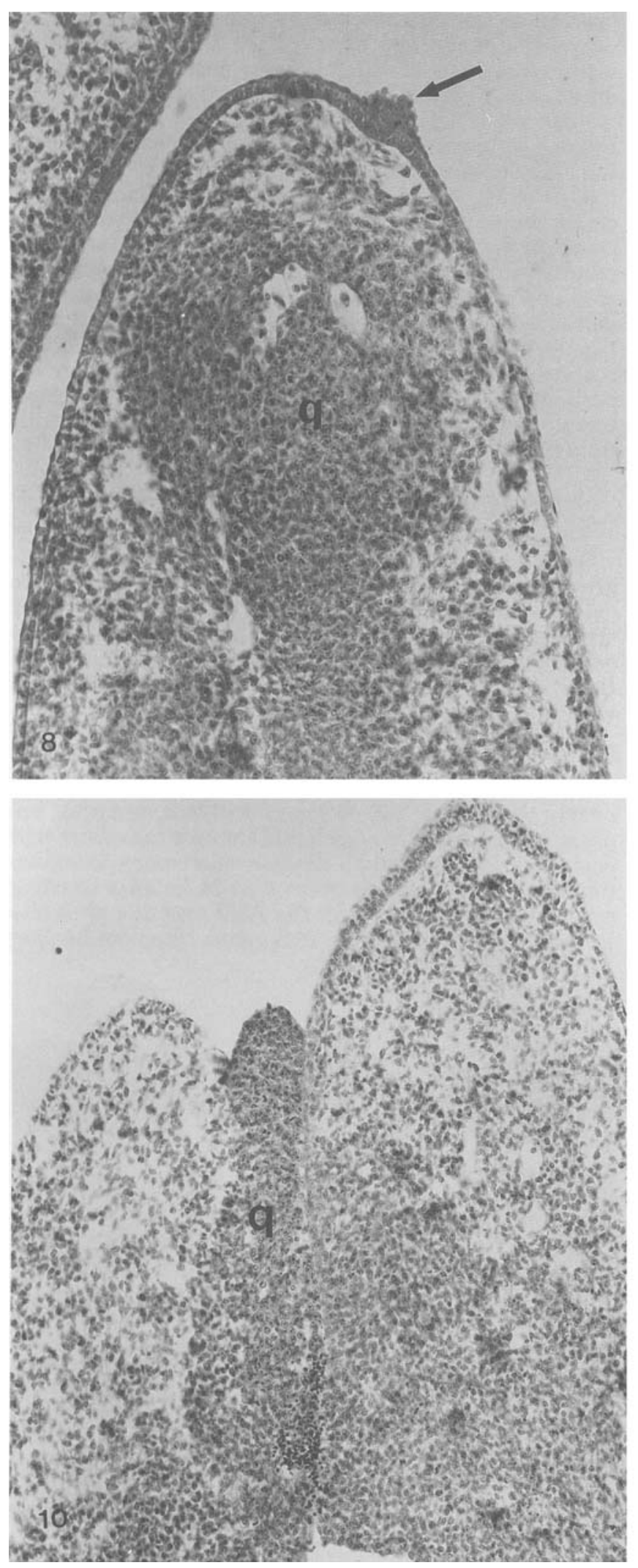
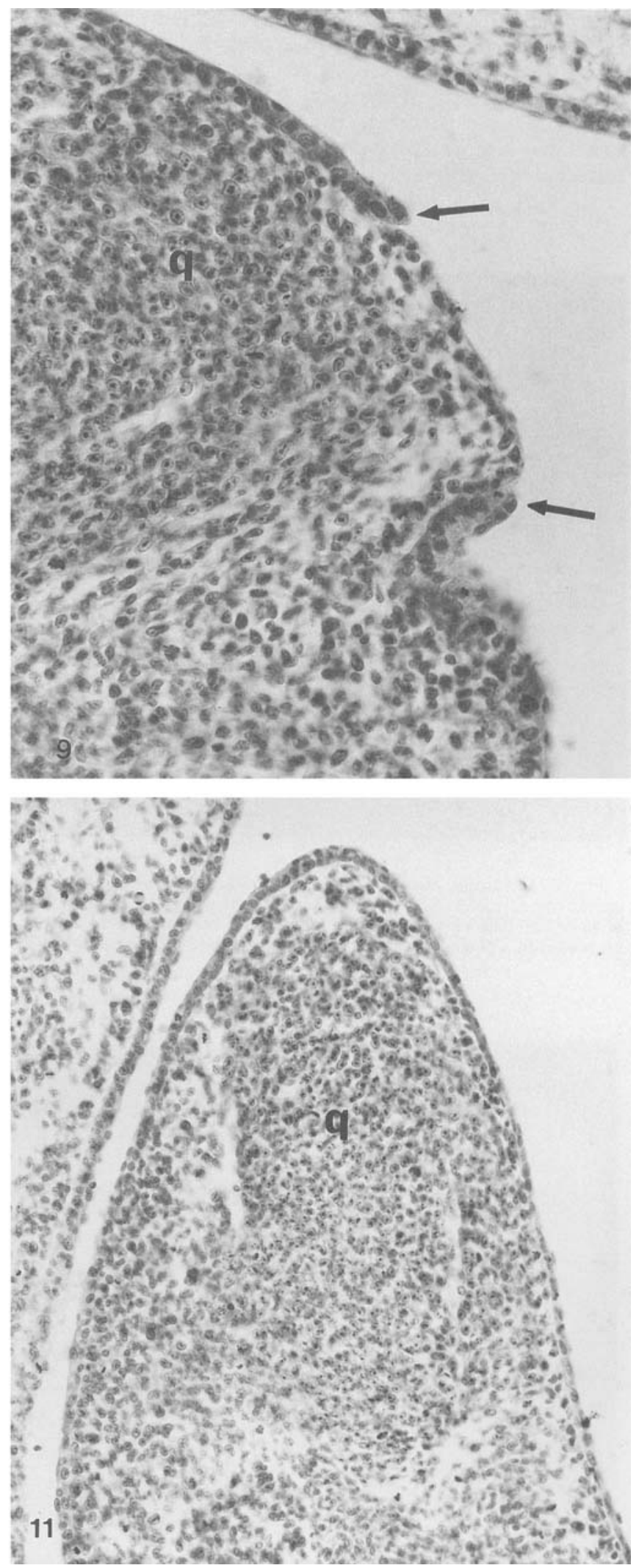
(Figs. 6, 7). In two embryos the AER appeared to be continuous, however, whether by approximation or actual regrowth, over the graft site. Ectodermal healing was incomplete in 11 of 17 embryos examined by light microscopy at 22-28 hr. The ectoderm was thickened at its healing edges in eight of these embryos. Of the six embryos with complete ectodermal healing over the graft, the ectoderm was thickened asymmetrically in two cases. However, this thickening did not resemble a typical AER (Fig. 8) and was possibly related to the meeting of migrating sheets of ectoderm with thickened edges.

Five serially sectioned experimental embryos were examined at $41 \mathrm{hr}$. In two embryos the healed ectoderm was not thickened; in two the healed ectoderm was thickened in a disorganized asymmetrical fashion near the graft; and in the remaining embryo an ectoderm with a thickened edge had not yet completely covered the wound (Fig. 9).

By $24 \mathrm{hr}$, the ectoderm had healed in all control (A-to$\mathrm{A}$ and P-to-P grafts) embryos examined by light microscopy (seven embryos) and SEM (four embryos). Of the latter, there was a gap in the AER across the graft in two cases. The other two cases were indeterminate.

\section{Distribution of quail cells within host limbs}

In experimental (A to $P$ ) embryos fixed for light microscopy at 3,6 , and $8 \mathrm{hr}$ post-operatively (12 embryos total), the implanted quail cells remained as a discrete, compact clump, with little mixing with chick cells at the graft-host margins. Necrotic mesodermal and red blood cells were occasionally found on the exterior surface of the graft, but the majority of the quail cells appeared to be healthy. There was no detectable difference in the histological appearance of the experimental embryos and 14 control ( $A$ to $A$ and $P$ to $P$ ) embryos fixed at 2, 6, and $12 \mathrm{hr}$ postoperatively (Fig. 10).

Serial sections of experimental (A to P) embryos fixed at 16,22 , and $28 \mathrm{hr}$ (18 embryos total) showed that the quail cells remained as a compact clump, with slight mixing of chick and quail cells at the graft-host interfaces. A short, narrow strip of quail cells extended distally from the main cluster of cells just beneath the ectoderm in two embryos. In the majority of these em-

Fig. 8. Feulgen-stained transverse section through a chick wing bud fixed $28 \mathrm{hr}$ after a wedge of pelleted, dissociated anterior quail wing mesoderm was implanted in a posterior slit. The ectoderm was healed and is thickened in an irregular fashion (arrow) near the apex of the bud. In this section, the grafted quail cells $(q)$ are prominently repre sented in the wing bud tissues. $\times 200$.

Fig. 9. Feulgen-stained transverse section through a chick wing bud fixed $41 \mathrm{hr}$ after a graft of pelleted, dissociated anterior quail (q) wing mesoderm was implanted into a posterior slit. The ectoderm has not healed completely (between arrows) and is slightly thickened at one edge. $\times 325$.

Fig. 10. Feulgen-stained sagittal section through a chick wing bud fixed $6 \mathrm{hr}$ after a wedge of pelleted, associated posterior quail wing mesoderm was implanted into a posterior slit. The quail cells (q) remain as a coherent, compact mass. $\times 150$.

Fig. 11. Feulgen-stained transverse section through a chick wing bud fixed $22 \mathrm{hr}$ after a wedge of pelleted, dissociated anterior quail wing mesoderm was implanted into a posterior slit. Most of the quail cells (q) are found in a pre-cartilage condensation, which is surrounded by blood vessels and some additional quail cells. $\times 195$. bryos (14/18), most of the quail cells were arranged in the compact, whorled configuration typical of a pre-cartilage condensation. This was usually surrounded by quail cells with the appearance of loose, undifferentiated mesenchyme. Blood and/or lymphatic vessels were usually found within the graft tissue and often also separated the quail cells within the pre-cartilage condensation from those within the surrounding mesenchyme (Fig. 11).

At $41 \mathrm{hr}$ post-operatively, the mixing of quail and chick cells at the graft-host margins was slightly increased, but the majority of the quail cells were contained within a discrete pre-cartilage condensation interdigitated with and surrounded by blood and/or lymphatic vessels (eight embryos examined). Additional quail cells in the form of undifferentiated mesenchyme surrounded the pre-cartilage condensation.

By $24 \mathrm{hr}$ and at later time points, the quail cells in the control embryos seemed to be more spread out than those in the experimental embryos. In $\mathrm{P}$ to $\mathrm{P}$ controls, the quail cells were typically found in the region of the elbow and proximal ulna and often extended into the distal humerus and distally along the posterior side of the ulna. The quail cells were found within the normal cartilage and pre-cartilage of the host wing bud, and in the perichondrium, loose mesenchyme, and dermis nearby (seven embryos examined).

In the $\mathrm{A}$ to $\mathrm{A}$ controls, the quail cells were found in the region of the anterior side of the proximal or distal humerus. Some quail cells were arranged in a whorled configuration within the host pre-cartilage, while others formed part of the loose mesenchyme and dermis (four embryos examined).

\section{Tissue Culture Studies}

Cultured pieces of quail mesoderm were analyzed histologically in order to determine the state of differentiation at times when pieces were implanted into chick hosts. For the sake of consistency in the tabulation of results, the cultured mesoderms were placed into one of four categories on the basis of their alcian-blue-staining properties. The categories are defined as follows: 1) no detectable alcian blue staining, 2) faint traces of disconnected alcian blue stain in the intercellular matrix, 3) various sized networks of light-to-moderate intensity of alcian blue staining, and 4) areas of histologically evident pre-cartilage and cartilage within the cultured mesoderm.

We commonly found evidence of cellular degeneration in 1-day cultures of all types of mesoderm (Fig. 12). By 2 days, the degeneration was, for the most part, resolved; further degenerative changes were not seen in our cultures (Fig. 13).

Figures 14 and 15 show the results of culturing both intact and dissociated-pelleted pieces of anterior and posterior quail wing mesoderm. There was a strong similarity among all four groups in the time course of cartilage differentiation. During the first day, there was minimal deposition of alcian-blue-staining matrix (5 of 82 total cases). By the second day faint flecks of bluestaining matrix material were seen in the majority of tissues ( 80 of 102 total cases). At 3 days a prominent network of stained matrix was seen in one area of most cultures. In only $21 \%$ of 3-day cultures (20 of 96 cases) was alcian blue staining minimal or absent. Histologi- 


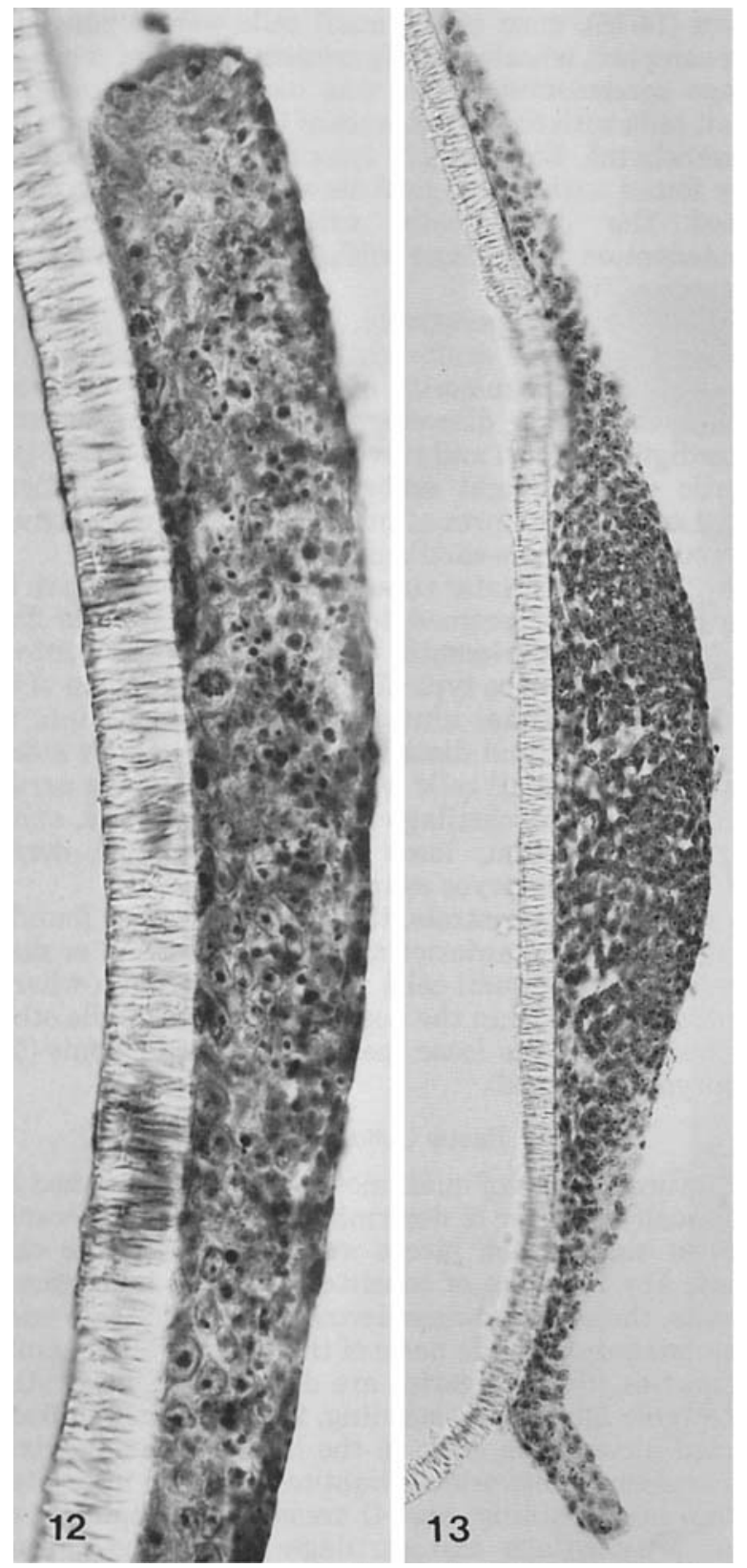

Fig. 12. Piece of pelleted, dissociated anterior quail wing bud mesoderm 1 day after having been placed into tissue culture. Scattered among the viable cells are remnants of degenerated cells. Alcian blue and hematoxylin stain. $\times 228$.

Fig. 13. Piece of pelleted, dissociated anterior quail wing bud mesoderm 3 days after having been placed into tissue culture. The tissue is undergoing early chondrogenic changes in the raised central area. At this time there is minimal evidence of cell degeneration. Alcian blue and hematoxylin stain. $\times 108$.

cally recognizable cartilage was the rule in 4- and 5-day cultures.

The major difference among the groups of cultured mesoderm was a difference in thickness between anterior (both intact and dissociated) and posterior mesoderm. By the second day in culture, anterior mesodermal pieces typically assumed a gross configuration similar to that of a fried egg, with a raised chondrogenic central area and a flattened periphery. In contrast, cultured posterior mesoderm exhibited a strong tendency to flatten out into broad, extremely thin sheets. Despite the thinning out, the time course of chondrogenesis in posterior mesoderm cultures was surprisingly similar to that of anterior cultures. However, the amount of cartilage formed in posterior pieces was often less than that formed in anterior pieces. An examination of Figure 15 reveals that a small proportion of cultured posterior mesoderm failed to form cartilage.

\section{Size of Cartilaginous Structures}

The supernumerary cartilaginous structures that developed following grafts of anterior quail mesoderm to posterior slits in host wing buds were significantly larger $(P<.01)$ than the cartilage nodules that developed in the in vitro cultures of anterior or posterior mesoderm. The mean size (largest dimension) of the supernumerary cartilage structures that formed by 5 days after the anterior-to-posterior operation was $1.8 \mathrm{~mm}(\mathrm{SE}=0.244$ $\mathrm{mm}, \mathrm{No} .=13$ ). For 5-day cultures of anterior mesoderm, the mean size of the cartilage nodules was $0.192 \mathrm{~mm}$ $(\mathrm{SE}=0.025$, No. $=12$ ); for posterior mesoderm the mean size of the nodules was $0.137 \mathrm{~mm}(\mathrm{SE}=0.009$ $\mathrm{mm}$, No. $=13$ ). The difference between the size of the nodules in the anterior and posterior cultures was statistically significant $(P<.025)$.

\section{DISCUSSION}

From previous work (Carlson, 1984a) we know that high percentages of supernumerary cartilage form when fresh anterior quail mesoderm from stage 17-24 donors is grafted into posterior slits. The incidence of supernumerary cartilage formation is not significantly altered when the donor mesoderm is cultured in vitro for 1-4 days prior to being grafted to a posterior location (Carlson, 1984b). These results were interpreted as a positionally related control of cartilage differentiation; supernumerary cartilage is stimulated to develop when anterior mesoderm is placed in a posterior position, but not when anterior or posterior mesoderm is placed in homologous positions. The culture experiments show that this property does not depend on a continuing interaction with or influence from the entire wing field upon the donor mesoderm.

The present study shows that the ability to participate in the positionally related differentiation of supernumerary cartilage does not require the grafted mesodermal cells to remain in the same topographical relationship to one another, as they were in the wing bud of the donor. It also demonstrates that mesodermal cells do not need to be in constant contact with one another, via gap junctions or other mechanisms, to retain this property. The extracellular matrix surrounding the cells of the donor mesoderm is largely removed by enzymatic digestion when the mesoderm is dissociated; therefore the established matrix probably does not serve as the sole repository of positional memory or positional value in these cells. It is quite possible, however, that after the dissociated mesodermal cells are pelleted and either cultured or implanted, morphogenetic (positional) information is expressed through the reestablishment of the extracellular matrix or a network of gap junctions. 

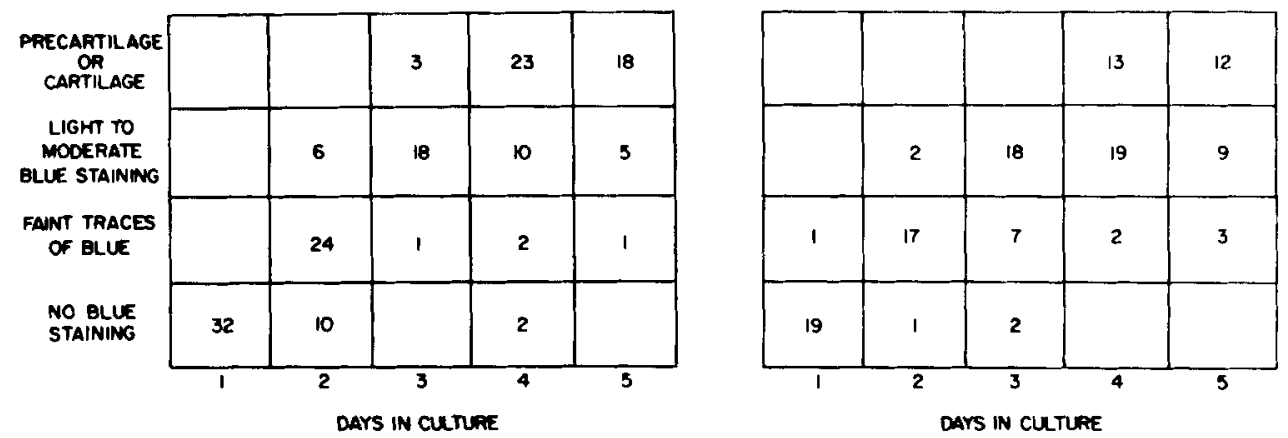

Fig. 14. Chart showing the alcian blue staining properties over time of pieces of intact anterior (left) and posterior (right) quail wing bud mesoderm (stages 21-23) in culture. Numbers in the squares refer to numbers of cultures examined.
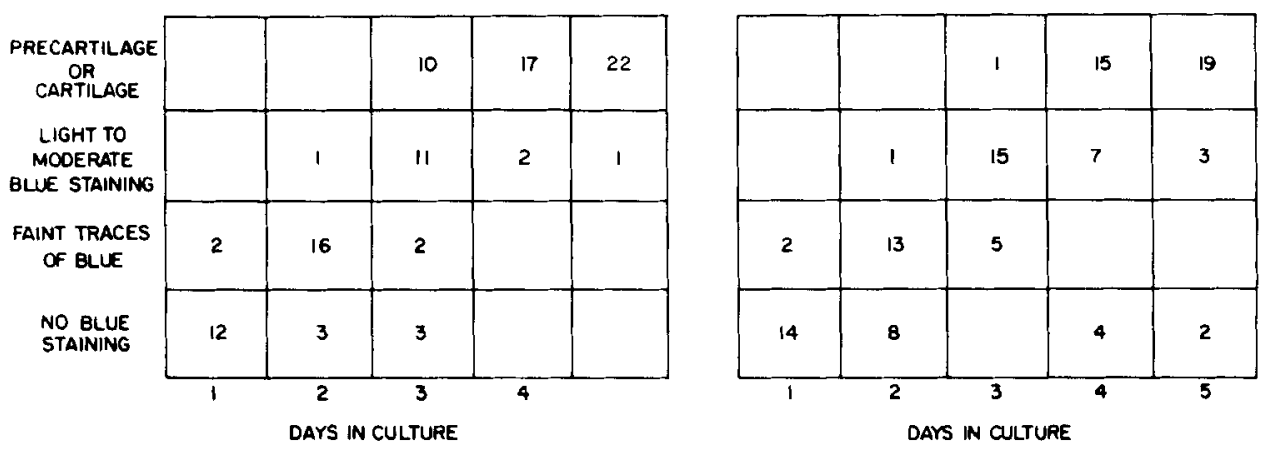

Fig. 15. Chart showing the alcian blue staining properties over time of pieces of pelleted, dissociated anterior (left) and posterior (right) quail wing bud mesoderm (stages 21-23) in culture. Numbers in the squares refer to numbers of cultures examined.

The results of the grafting experiments with cultured pellets of dissociated mesoderm demonstrate that the ability of the donor mesoderm to participate in the positionally related differentiation of supernumerary cartilage does not require a continuing influence from the limb field. Like intact mesoderm, cultured dissociated mesoderm retains the ability to participate in the formation of supernumerary cartilage for longer than it would if it remained in situ in the wing bud of the donor (Carlson, 1984a).

Pieces of anterior mesoderm that had been pelleted and implanted into posterior slits immediately after dissociation produced supernumerary cartilage in $74.3 \%$ of the cases; pelleted dissociated mesoderm that had been cultured in vitro for 1 or 2 days prior to implantation produced $95.5 \%$ and $93.8 \%$ incidence, respectively, of supernumerary cartilage. In all cases, the percentage of supernumerary cartilage produced following the corresponding control operations (anterior-to-anterior and posterior-to-posterior) was very low.

There are several possible reasons for the lower percentage of supernumerary cartilage seen after implanting pieces of pellet from freshly dissociated mesoderm. One possible explanation is purely technical. Freshly pelleted mesodermal cells do not adhere tightly, and a significant number of donor cells may have been lost after they were implanted in the host wing. Another possibility is that the mesodermal cells sustained acute damage from the enzymatic dissociation and that the damage compromised their ability to play a role in the formation of supernumerary cartilage when they were grafted immediately after dissociation. It might be expected that such damage would be repaired (perhaps via synthesis of membrane components) in cell pellets that were cultured in vitro prior to being implanted. But because the incidence of supernumerary cartilage was significantly higher following the experimental operations using freshly dissociated mesoderm than in the corresponding controls, we suggest that such repair is not essential for the positional response assayed here.

The high percentages of supernumerary cartilage formed after anterior-to-posterior grafts of 1- and 2-day cultured mesoderm that had been previously dissociated and pelleted are similar to those observed after grafting of 1-and 2-day cultured intact mesoderm. The data on longer-term cultures of both intact and dissociated mesoderm are more difficult to interpret because the incidence of supernumerary cartilage formation decreased after the experimental operations and increased after the control operations. Therefore, the 1- and 2-day cultured mesoderm would be the most useful for future experiments because it provides a high "signal-to-noise" ratio.

The tissue culture studies showed that the time course of cartilage differentiation, as indicated by the alcianblue-stained extracellular matrix, was very similar in 
intact and dissociated-pelleted pieces of anterior and posterior mesoderm. Mesoderm cultured for 3 days typically showed an extensive network of alcian-blue-staining matrix. After 4 days in culture, most of the mesoderm formed histologically recognizable pre-cartilage or cartilage. Thus the decreased incidence of supernumerary cartilage following grafts of 3- and 4-day cultured anterior mesoderm to posterior slits $60 \%$ and $65 \%$, respectively, compared with $93.8 \%$ for 2-day cultures) might be explained if the onset of cartilage differentiation inhibited the ability of the donor mesoderm to stimulate more extensive, positionally related chondrogenesis. This possibility is based upon the assumption that the pre-cartilage and small cartilage nodules detected by alcian-blue-staining in sections of cultured mesoderm would not be detected in the whole-mount preparations of wings stained for cartilage with Victoria blue B dye.

It is important to note that ectopic chondrogenesis was quite minimal following grafts of 3- to 4-day cultured anterior or posterior mesoderm to homologous locations in host wings. It appears that the tendency toward chondrogenesis that is characteristic of cultured mesoderm can be suppressed when the tissue is grafted at a homologous anterior-posterior level. This was also observed by Carlson (1984b) when cultured wedges of intact mesoderm were implanted in host wings. Again, we cannot be sure whether the small cartilage nodules that presumably had already formed in the cultured donor mesoderm would be detected in the whole-mount preparations stained with Victoria blue B dye.

The supernumerary cartilaginous structures that developed 5 days after pieces of anterior mesoderm were grafted to posterior slits were significantly larger than the cartilage nodules that formed in 5-day in vitro cultures of anterior or posterior mesoderm. In addition, the cartilage structures that developed in vivo were often more complex in morphology (rods or spurs, or irregular nodules) than the small nodules of cartilage that developed in vitro. Therefore, the increased incidence of supernumerary cartilage formation following anterior-toposterior grafts is not caused merely by a response of the donor cells to being removed from their normal environment (analogous to placing the mesoderm in culture in vitro. It is in some way stimulated by the apposition of anterior and posterior cells. This idea is supported by the results of control experiments done by Carlson (1984a); grafts of anterior or posterior quail wing bud mesoderm grafted into the flank of host chick embryos produced ectopic cartilage in only $22.2 \%$ and $31.2 \%$ of the cases, respectively.

The time course and morphology of ectodermal healing was revealed by scanning electron microscopy and histological analysis of embryos in which freshly dissociated mesoderm had been implanted in the wing bud. In both experimental (anterior-to-posterior) and control (anterior-to-anterior and posterior-to-posterior) embryos, the ectoderm usually healed by $22-28 \mathrm{hr}$ after the operation, although the healing was sometimes incomplete at $41 \mathrm{hr}$ post-operatively. There was very little evidence that the AER was reestablished over the graft site. Although the ectoderm appeared to be thickened in the region of the graft in a few scanning electron micrographs, the histological analysis showed that this thickening was asymmetrical and did not resemble a typical
AER. In many embryos in which ectodermal healing was incomplete, the ectoderm was thick and irregular at its healing margins. Therefore it is likely that the asymmetrical thickening seen in healed ectoderms was merely "left over" when the thick advancing edges of the healing ectoderm met and thus that it was not a true AER.

The formation of the AER has not previously been examined systematically in experiments in which mesoderm (without its overlying ectoderm) was implanted in host wing buds (Fallon and Thoms, 1979; Carlson, 1984a,b), but Saunders (1948) demonstrated that the AER was not regenerated when it was removed from chick wing buds. When anterior mesoderm (chick or quail) plus the overlying ectoderm is grafted to a posterior slit in a host chick wing bud, recognizable supernumerary digits usually develop (Iten and Murphy, 1980; Carlson, 1984a,b). However, only rods and nodules of supernumerary cartilage, and never recognizable digits, develop when anterior mesoderm alone is grafted. This can probably be explained by the inability of the AER to form over the graft, since the AER is essential for outgrowth of the subjacent mesoderm (Saunders, 1948; Summerbell, 1974).

There appeared to be no difference in the distribution and arrangement of quail (graft) cells within the host wing buds in control and experimental embryos fixed during the first $12 \mathrm{hr}$ after the operation. In all cases, the quail cells remained as a discrete, compact cluster, with little or no mixing with the surrounding chick cells. However, by $24 \mathrm{hr}$ post-operatively and at later time points, the quail cells appeared to be slightly more dispersed in the control embryos than in the experimental embryos. This can probably be explained by the observation that a large proportion of the quail cells in experimental embryos were usually included within a single pre-cartilage condensation, whereas the majority of the quail cells in control embryos were usually found within the normal soft tissues of the host limb.

We do not know whether the positionally related differentiation of supernumerary cartilage demonstrated by Carlson $(1984 a, b)$ in intact mesoderm, and here in dissociated and pelleted mesoderm, represents the same type of positional effect as shown by the development of supernumerary digits when anterior mesoderm plus the overlying ectoderm is grafted to posterior slits in host wing buds (Iten and Murphy, 1980). The supernumerary cartilage may be stimulated to develop via local interactions among cells of disparate positional values (anterior and posterior). Alternatively, the cells of the graft may be stimulated to differentiate into supernumerary cartilage in response to the influence of the surrounding polarizing zone of the host. A third possibility must be considered: grafted quail mesoderm may have a greater intrinsic tendency to differentiate into cartilage, or quail mesoderm may be more responsive to positional differences than chick mesoderm. Grafts of chick anterior mesoderm to posterior sites in chick wing buds have resulted in 9\% (Fallon and Thoms, 1979) to 36\% (Carlson, 1984a) supernumerary structures. Comparable anterior-to-posterior grafts in the quail wing bud or grafts of chick mesoderm into quail wing buds have not been reported.

In a recent abstract, Suzuki and Ide (1986) reported that in vitro chondrogenesis of chick leg bud mesoderm 
was stimulated when pieces of anterior and posterior mesoderm were pinned together and cultured. In contrast, large cartilaginous masses did not form when anterior or posterior mesoderm was cultured alone or when anterior-anterior or posterior-posterior combinations were cultured. In these experiments the ectoderm was left on the mesoderm; the enhanced chondrogenesis in anterior-posterior combinations was inhibited when the ectoderm was removed. The results of these experiments may represent a positional effect similar to the one assayed in vivo by Carlson $(1984 \mathrm{a}, \mathrm{b})$ and in the present study.

\section{ACKNOWLEDGMENTS}

Supported by NIH grant HD-17228 to B.M.C. and an NIH predoctoral training grant fellowship (T32 HD 07274) to K.M.S. The authors wish to thank Jean Carlson and Cindy Fabricius-Segal for technical assistance, John Beckerman for photography, William Brudon for artwork, and Janis Beard for secretarial assistance.

\section{LITERATURE CITED}

Carlson, B.M. 1984a The formation of supernumerary structures after grafting anterior quail wing bud mesoderm of various ages into chick wing buds. Dev. Biol., 101:97-105.

Carlson, B.M. 1984b The preservation of the ability of cultured quail wing bud mesoderm to elicit a position-related differentiative response. Dev. Biol., 101:106-115.

Carlson, B.M., B.K. Simandl, K.M. Stocker, T.G. Connelly, and J.F Fallon 1986 A method for the combined gross skeletal staining and Feulgen staining of embryonic chick tissues. Stain Technol., 61:2731.

Fallon, J.F., and G.M. Crosby 1977 Polarizing zone activity in limb buds of amniotes. In: Vertebrate Limb and Somite Morphogenesis.
D.A. Ede, J.R. Hinchliffe, and M. Balls, eds. Cambridge University Press, Cambridge, pp. 55-69.

Fallon, J.F., and S.D. Thoms 1979 A test of the polar coordinate model in the chick wing bud. Anat. Rec., 193:534.

French, V., P.J. Bryant, and S.V. Bryant 1976 Pattern regulation in epimorphic fields. Science, 193:969-981.

Hamburger, V., and H.L. Hamilton 1951 A series of normal stages in the development of the chick embryo. J. Morphol., 88:49-67.

Hill, A.B. 1966 Principles of Medical Statistics. Oxford University Press, New York.

Iten, L.E. 1982 Pattern specification and pattern regulation in the embryonic chick limb bud. Am. Zool., 22:117-129.

Iten, L.E., and D.J. Murphy 1980 Pattern regulation in the embryonic chick limb: Supernumerary limb formation with anterior (nonZPA) limb bud tissue. Dev. Biol., 75:373-385.

Javois, L.C and L.E. Iten 1981 Position of origin of donor posterior chick wing bud tissue transplanted to an anterior host site determines the extra structures formed. Dev. Biol., 101:329-342.

LeDouarin, N. 1973 A biological cell labeling technique and its use in experimental embryology. Dev. Biol., 30:217-222.

Lev, R., and S.S. Spicer 1964 Specific staining of sulfate groups with Alcian blue at low pH. J. Histochem. Cytochem., 12:309

Saunders, J.W., Jr. 1948 The proximo-distal sequence of origin of the parts of the chick wing and the role of the ectoderm. J. Exp. Zool., 108:363-404

Saunders, J.W., Jr., and M.T. Gasseling 1968 Ectodermal-mesenchymal interactions in the origin of limb symmetry. In: EpithelialMesenchymal Interactions. R. Fleischmajer and R.E. Billingham, eds. Williams \& Wilkins, Baltimore, pp. 78-97.

Summerbell, D. 1974 A quantitative analysis of the effect of excision of the AER from the chick limb bud. J. Embryol. Exp. Morphol., 32:651-660.

Suzuki, H.R., and H. Ide 1986 Heterogeneity and interaction between fragmented avian limb buds in organ culture. Zool. Sci., 3:1071.

Tickle, C., D. Summerbell, and L. Wolpert 1975 Positional signalling and specification of digits in chick limb morphogenesis. Nature (Lond), 254:199-202.

Wolpert, L. 1969 Positional information and the spatial pattern of cellular differentiation. J. Theor. Biol., 25:1-47.

Wolpert, L. 1971 Positional information and pattern formation. Curr Top. Dev. Biol., 6:183-224. 